AperTO - Archivio Istituzionale Open Access dell'Università di Torino

\title{
Evaluation of the effectiveness of neuromuscular electrical stimulation after total knee arthroplasty: a meta-analysis
}

\section{This is a pre print version of the following article:}

Original Citation:

Availability:

This version is available http://hdl.handle.net/2318/1654463

since 2019-09-30T00:22:31Z

Published version:

DOI:10.1097/PHM.0000000000000847

Terms of use:

Open Access

Anyone can freely access the full text of works made available as "Open Access". Works made available under a Creative Commons license can be used according to the terms and conditions of said license. Use of all other works requires consent of the right holder (author or publisher) if not exempted from copyright protection by the applicable law. 
Title

Evaluation of the effectiveness of neuromuscular electrical stimulation after total knee arthroplasty: a meta-analysis.

\section{Abstract}

Aim: to analyze and evaluate the efficacy of the use of the neuromuscular electrical stimulation (NMES) after total knee arthroplasty.

Methods: a systematic review of randomized controlled trials identyfied through MEDLINE, PubMed, Cochrane Library, and PEDro. The PICOS approach was used to formulate the research question. The criteria for inclusion and exclusion has been defined in advance. Controlled terms, and Boolean operators have been used to increase the sensitivity and specificity of the search. "Neuromuscular electrical stimulation" and "total knee arthroplasty" has been used as keywords. The overall risk of bias was determined according to the following domains: random sequence generation, concealment, blinding mass of participants and staff, commissioning blind assessment results, the presence of incomplete data and loans received.

Results: out of the 33 identified studies, six were included in the meta-analysis (496 participants). In these studies, one group of patients followed a rehabilitation protocol (controlgroup) and the other followed a rehabilitation program plus a session of neuromuscular electrical stimulation (NMES-group). Patients from the NMES-groups got the best scores (Up and Go Test, Stair-Climbing Test and Walk Test). The benefits from NMES were strong in the first postoperative weeks / months and gradually diminished.

Conclusions: there is scientific evidence to sustain that the NMES allows a better functional recovery following the total knee arthroplasty, especially in the first period. This is particularly true for individuals with a severe lack of muscular activation of neuronal-type.

\section{Key words}

Neuromuscular Electrical Stimulation Total Knee Arthroplasty

\section{Introduction}

Total knee arthroplasty (TKA) is effective for relieving pain and improving function in patients with end stage arthritis of the knee [1-5]. However, a complete functional recovery may not be achieved, with up to $20 \%$ of patients with a certain degree of dissatisfaction after TKA [6, 7]: pain, stiffness and weakness are among the leading causes of this dissatisfaction [8]. Weakness in particular, which is often already present before surgery, still remains after surgery for long time, in fact differences have been shown between subjects after TKA surgery and healthy adults of the same age [9]. These functional deficits may have major consequences for the patients from a clinical standpoint: reduced walking speed and balance, difficulties with stairs and increased risk of falls [10].

Neuromuscular electrical stimulation (NMES) has been proposed as an adjunct to traditional rehabilitation programs for patients after TKA, especially for those with deficit of the voluntary activation of the muscle and postoperative weakness [11,12]. The contractions induced by electrical stimulation can lead to a more efficient muscle training with a greater and more selective recruitment of type II muscle fibers than voluntary contractions [13, 14]. In addition, the inputs generated by NMES may facilitate plastic changes in the networks of 
the senso-motor neurons at the level of the central nervous system, thus leading to a strengthening of the signals and pathways dedicated to the muscle control and strength [15, $16]$.

The primary purpose of this meta-analysis was to analyze the randomized controlled trials reported in literature that compared the results of a traditional rehabilitation program after TKA with a rehabilitation program implemented with the neuromuscular electrical stimulation.

\section{Materials and Methods}

Literature search strategies

The methodology of this study complies with PRISMA guidelines of 2009 [17]. The research has been conducted with Electronic databases (MEDLINE, PubMed, Cochrane Library, PEDro) and Bibliographies of identified studies and review, from June 2000 to June 2016. Within the literature search on Pubmed, terms or "MeSH terms" and Boolean operators were employed such as keywords, to increase the sensitivity and specificity of the search. Keywords used for research were: "neuromuscular electrical stimulation” and "total knee arthroplasty". The PICOS approach has been used to formulate correctly the questions (Patients, Intervention, Comparator, outcomes, Study design) [17].

\section{Inclusion criteria}

The studies included in the meta-analysis were required to meet the following criteria: a) randomized controlled trials evaluating the efficacy of NMES in patients undergoing unilateral TKA; b) rehabilitation with NMES for a minimum duration of 4 weeks; c) presence of a control group; d) clinical outcomes defined a priori. "English language" and "the last 16 years" have been established as limits for the research. Table 1 reports inclusion and exclusion criteria.

\section{Data collection}

A standardized data extraction was used for data collection and Excell program was used for archiving and analysis. The characteristics of the selected studies have been extracted, included: publication date, demographic characteristics of participants (mean age; percentage of females; Mean Body Mass Index - BMI), intervention studies, sample sizes (number of subjects in the intervention and control groups), the study design, the type of action taken (commencement, duration and frequency of treatment; intensity, frequency, duty cycle and pulse duration; electrode size), outcomes used by individual studies and the period of follow up.

Analyses of potential bias

The overall risk of bias was determined according to the following domains: generate random sequence, concealment of allocation, blind setting of participants and staff, blind assessment of the results, incomplete data and funding received.

Statistical analysis

The synthesis of the quantitative data was performed using electronic tables in Microsoft Excell specially designed for creating meta-analysis. The tables were made following the guidelines provided by the Creative Commons Attribution License and published on Bio Med Central Research Notes [18].

When data on at least three studies were available, a meta-analysis was carried out. 
Heterogeneity across studies was quantified using I-squared statistics and tested with Q Cochran's statistics. Given the small number of studies the pooled mean difference was estimated along with the corresponding 95\% confidence intervals (CI), using the fixed-effect model weighting scheme. To assess publication bias, funnel plot techniques, Begg's rank test and Egger's regression test were intended to be used, as appropriate given the known limitations of these methods. Statistical analyses were carried out with R, version 3.2.5

\section{Results}

Studies selection

The PRISMA flow diagram reports the assessed items and the reasons for exclusions (Figure 1): from the starting 33 studies identified through the search in the databases, only six randomized controlled trials reflected the inclusion criteria and were included in the metaanalysis [19-24]. Twenty-two studies had been excluded because did not match inclusion criteria. The study of Stevens-Lapsley et al. of 2012 [25] was excluded because it was a substudy of the work done by the same team [22]. Similarly, the study of Laufer et al. of 2010 [26] was excluded as it formed a sub-study of the work done by Petterson et al. [23]. Three case reports [11, 12, 27], a case series [28] and a clinical trial [29] have been excluded because evaluated the use of NEMS only before surgery and not after.

\section{Characteristics of the studies}

Characteristics of participants. The size of the studies included ranged from 30 to 200 participants involving a total of 496 patients. Approximately half of the patients followed a standard rehabilitation protocol (control-group) and the other part followed a rehabilitation program plus a session of neuromuscular electrical stimulation (NMES-group). A number of 250 patients were randomized in NMES-Group, while 246 in the control-group; follow-up periods ranged between 4 and 52 weeks. Overall, the median age was 67.2 years, with $69.9 \%$ of women and a mean BMI of 29.4. In most of the trials included in the meta-analysis, patient selection was controlled with particular attention to the criteria listed above, including only relatively healthy subjects who had knee replacement surgery for unilateral arthritis of the knee. In these studies, patients who had significant pain in other joints of the lower limbs, other comorbid conditions (uncontrolled diabetes, etc) or cardiovascular diseases have been excluded. An exception to the criteria mentioned above, was the study of Levine et al. [21] where patients with knee arthritis and bilateral pain were included. In this study, the patients with comorbidities such as epilepsy, lower limb ischemia, or decreased cognitive function were not included. Table 2 reports the demographic of the participants and the characteristics of the studies.

Characteristics of the procedures. The characteristics of the neuromuscular electrical stimulation among the various studies were similar, although some variations of parameters were present. The frequencies of stimulation in all studies were sufficient to induce a tetanic contraction of the muscle, with variations between 40 and 100 beats per second (Hz). The duration of the impulse applied to the muscle stretches between 250 and $400 \mu$ s. All studies used the maximum intensity tolerated by the patient. On the contrary, the Duty cycle (ratio between the time when the machine dispenses the impulse and time of rest) showed considerable variations between different studies. In most studies, both groups (controlgroup and NMES-group) followed a rehabilitation protocol that included strength exercises,
Commentato [pb1]: Ecco la citazione se ti serve: R Core Team (2016). R: A language and environment for statistical computing. R Foundation for Statistical Computing, Vienna, Austria. 
flexibility and functional type workouts. The NMES-Groups performed in addition neuromuscular electrical stimulation sessions as shown in the Table 3.

Description of bias

Table 4 summarizes the quality and the risk of bias of all individual randomized controlled trials included in the meta-analysis. The overall quality of the included studies was acceptable, in particular if considering that all studies were randomized, that the quality of the data was good and complete, that this was not a drug therapies study and that is this kind of studies where blindness can be complicated. The study of Petterson et al. [23] had the lowest risk of bias: it was single blind study, which responded positively to all the questions examined, with the exception of concealment of allocation which was not mentioned. On the contrary, the study that proved a major risk of bias was that of Stevens-Lapsley et al. [22]: in this study, the evaluators were aware of the type of treatment received by participants and, in plus, this was the only study that received economic support.

In the meta-analysis, publication bias was not assessed as there were inadequate numbers of included trials to properly assess a funnel plot or more advanced regression-based assessments.

\section{Outcomes}

The absence of a defined protocol, involved that, among the various studies, many different outcomes were used to evaluate the efficacy of NMES. Table 5 reports all the outcomes described in the studies, divided into two groups. The first group of outcomes evaluated the physical performance of patients during the rehabilitation phase: aim of these outcomes was to describe appropriately the physical adaptation of the patients to the prosthesis and how this affects the everyday performances like walking, getting up, sitting, climbing stairs. The second group included outcomes whose primary purpose was to provide an estimate of the overall functionality of the knee. These are well-known questionnaires about social and emotional functions, pain and disability, and how these affect the subject's life (Table 5).

Analysis of the results

In the first study of Avramidis et al. [19] the participants, randomized in two groups (NMES and control), were assessed prior surgery and at 6 and 12 weeks after surgery. The NMESGroup presented a longest distances covered in the 3 minutes walking test (3MWT) than the control group with a statistically significant difference, both at $6(\mathrm{p}=0.0002)$ and at 12 weeks ( $p<0.0001$ ). On the contrary, the differences measured between the two groups regarding the Physiological Cost Index (PCI) and Hospital for Special Surgery Knee Score (HSS) were not statistically significant $(\mathrm{p}>0.2)$.

Petterson et al. [23] evaluated the participants pre-operatively and then at 3 and 12 months after surgery and did not show significant differences $(p>0.08)$ for all examined outcomes between the two types of interventions (physical exercises or physical exercises associated with NMES). These two groups of participants, however, showed better results than a third control group of 41 patients who followed a standard rehabilitation protocol ("standard of care"): the outcomes assessed at 12 months after the intervention showed a statistically significant difference (all p-value less than 0.01).

Stevens-Lapsley et al. [22] showed a statistically significant improvement $(\mathrm{p}<0.05)$ after 
3.5 weeks in favor of the NMES-Group regarding the following outcomes: strength of the hamstrings and of the quadriceps femoris, functional performance 6 minutes walking test (6MWT), Stair Climbing Test (SCT), Test Up and Go (TUG) and range of movement (ROM) for active extension. These differences were still present, although reduced after 52 weeks.

In the study of Avramidis et al. [20] the Knee Society Score (KSS) showed statistically significant differences in the functional and total score at 6 and 12 weeks after surgery. The other used outcomes (Oxford 12-item Knee Score and 3MWT) also emphasized that the two groups had statistically significant differences (with better results for the NMES-group) in the early postoperative period, which gradually declined over time.

Levine et al. 2013 [21], compared the two groups using six different outcomes: degree of extension, the degree of flexion, KSS (functional and related to pain), Western Ontario and McMaster Universities Arthritis Index (WOMAC) and TUG. The parameters were examined before surgery, at 6 weeks and then at 6 months. The study showed that the NMES-Group was not less than the control group for any considered outcomes, at both intervals.

In the study of Demircioglu et al. 2015 [24], VAS scores in both the first and third months after TKA were found to be significantly better in the NMES group than in the control group $(p=0.0)$, although knee flexion and extension ranges were significantly better only at the first-month follow-up visit $(p=0.05)$. The comparisons of WOMAC results at month 1 revealed that pain, stiffness, and total scores of the NMES-group was significantly better than those of control-group and significantly better physical function and SF-36 subscales, except mental health, were found for the NMES-group at the first month of follow-up.

In general, according to all studies, the neuromuscular electrical stimulation is a very simple physical therapy and allows a better functional recovery after total knee replacement surgery, at least in the first months.

\section{Discussion}

The origin of the weakness of the quadriceps muscle after TKA is multifactorial: the trauma of the surgery, factors associated with the pre-existing knee arthritis, general factors such as obesity, morbidities and age can all be cause of weakness. The mechanism that underlies the weakness can be of muscular nature (eg. atrophy, decrease in the number and size of muscle fibers) or related to neuronal reasons (eg. reduced voluntary muscle activation). The contribution of the neuronal type seems to be prevalent respect to the muscular one, especially in the first postoperative weeks after total knee replacement; on the contrary, after approximately 4-5 months the muscular component takes over [10]. Since weakness can be a severe impairment for the recover after TKA, NMES has been proposed in addition to standard rehabilitation programs.

This meta-analysis included six randomized controlled trials performed from 2003 to 2015, which together collected data on 496 patients undergoing total knee arthroplasty. All studies evidenced a deficit of strength and functionality in the first period after surgery, compared with data collected prior to the surgery: this was an effect of surgery itself. These deficits 
receded during the follow-up, also in response to the rehabilitative treatment and after only 6 months the results were already better than before surgery. All patients had excellent functional outcomes and physical performances, thus confirming the good results achieved at present with total knee arthroplasty and the subsequent rehabilitation.

Data analysis showed no inferiority of the rehabilitative treatment with neuromuscular electrical stimulation compared with physical therapy alone. Must be underlined the absence of a standardized common rehabilitative protocol in the five studies analyzed, that prevented a uniformity of outcomes. In relation to this issue of heterogeneity, outcomes were then divided into two categories: those based on tests that evaluated physical performance and those that evaluated the functionality of the prosthetic knee and the quality of life. This approach allowed to gather and analyze the homogeneous data properly and to extract the data that best reflected the real situation. On the other side, persons who during the rehabilitation period benefited from the neuromuscular electrical stimulation in addition to normal physical therapy, got higher scores compared with those who received physical therapy alone (in particular the tests that best expressed this difference were the TUG, SCT and 6MWT).

One of the findings of this meta-analysis was that the differences highlighted by one or several outcomes, were strong in the first few weeks/months after surgery and then gradually waned with passing of the time. This situation was related to the fact that in the early postoperative period there is an important deficit of muscle activation of neuronal type (or Central). For this reason, the use of a treatment that allows muscle activation device, such as NMES, was essential to prevent muscle atrophy and dysfunction.

Another evidence of the study concerns the optimal time for initiation of NMES. Petterson et al. [23] did not show any statistically significant difference between the NMES-group and the control-group, but this probably that was consequence of the fact that patients have begun treatment 4 weeks after surgery, while in the other five studies the NMES started after 24-48 hours. Adding treatments with NMES to conventional rehabilitation is therefore to be considered the more effective the more the shortfall of the muscular voluntary activation is pronounced, just like happened exactly during the early postoperative period.

A difference between the studies regarded the timing and the regularity of the application of the NMES. In the study of Petterson et al. [23] NMES was applied to the patients 2-3 times per week for approximately 15 minutes each time, while in the studies of Avramidis et al. [19, 20] and Stevens-Lapsley et al. [22] patients were instructed to apply by themselves. These different approaches led to widely differing treatment volumes between the studies. Clearly, the intensive treatment on a daily basis was more effective than the short bi-weekly sessions.

The intensity of the electrical stimulation also played a pivotal role in the effectiveness of the treatment. All studies used the maximum intensity tolerated by the patient; nevertheless, it has been noted that, in certain cases, the NMES may activate the nociceptive receptors, 
causing discomfort that can limit the effectiveness of the treatment. The use of electrodes with a large surface of skin contact can be a solution to this problem, because the possibility to reduce the current density (ratio of current and contact surface).

Most studies reported that the NMES allows a better functional recovery after total knee replacement surgery, particularly for individuals with a pronounced deficit of muscular activation of neuronal type. Also, no side effects has been correlated with the treatment with NMES, which has proven to be safe and free from risks to the health of the patient; finally, the application of NMES did not affect the integrity and the stability of the prosthesis. It is true that the differences tend to diminish after a certain time, but first is the control group that reaches the values of the NMES-group and not this one which loses the "advantage". Second, and most important, the faster is the recover the better are the result in the long time for the patients. The compliance to treatment was remarkable because the treatment was very simple. A cutaneous reaction due to the adhesive of the electrodes, with consequent discomfort reported by some individuals, has been the only side effects reported.

The major limitation identified in this meta-analysis was linked to reduced sample size of the studies (excluded the study of Petterson et al. [23]). Another limitation was represented by the heterogeneity of rehabilitation protocols, in particular as regards the setting of the NMES, but especially for the timing of commencement, duration and frequency of treatment. Also, for the studies of Avramidis et al. [19, 20] and for that of Stevens-Lapsley et al. [22] a blind assessment of the outcomes was not possible due to the reduced funds and therefore they incurred into a detection bias.

\section{Conclusions}

NMES after total knee arthroplasty offered several advantages: a) better and faster functional recovery, both from the point of view of the physical performance and of the joint function; b) increasing of quality of life, indices of disability, emotional and social functions. b) NMES is easy to apply and can be done daily by instructed persons, allowing a continuous and durable muscular training c) the use of NMES is safe and do not present any risk of injury secondary to treatment or implant damage.

\section{Conflicts of interest}

None: no benefits in any form have been received or will be received from a commercial party related directly or indirectly to the subject of this article.

\section{Caption}

Figure 1: The PRISMA flow diagram encapsulates the items assessed and the reasons for exclusions.

\section{References}

1] Bistolfi A, Lee GC, Deledda D, Rosso F, Berchialla P, Crova M, et al. NexGen® LPS mobile bearing total knee arthroplasty: 10-year results. Knee Surg Sports Traumatol Arthrosc 
2014;22(8):1786-1792.

2] Bistolfi A, Massazza G, Rosso F, Deledda D, Gaito V, Lagalla F, et al. Cemented fixedbearing PFC total knee arthroplasty: survival and failure analysis at 12-17 years. J Orthop Traumatol 2011;12(3):131-136

3] Buechel FF Sr. Long-term follow up after mobile-bearing total knee replacement. Clin Orthop Relat Res 2002;404:40-50

4] Hawker G, Wright J, Coyte P, Paul J, Dittus R, Croxford R et al. Health-related quality of life after knee replacement. J Bone Joint Surg Am 1998;80-A:163-173

5] Bistolfi A, Massazza G, Lee GC, Deledda D, Berchialla P, Crova M. Comparison of fixed and mobile-bearing total knee arthroplasty at a mean follow-up of 116 months. J Bone Joint Surg Am 2013;19-95(12):e83.

6] Beswick AD, Wylde V, Gooberman-Hill R, Blom A, Dieppe P. What proportion of patients report long-term pain after total hip or knee replacement for osteoarthritis? A systematic review of prospective studies in unselected patients. BMJ Open 2012;22-2(1):e000435.

7] Bourne RB, Chesworth BM, Davis A, Mahomed N, Charron K. Patient satisfaction after total knee arthroplasty: who is satisfied and who is not? Clin Orthop Relat Res 2010;468(1):57-63

8] Escobar A, Quintana JM, Bilbao A, Aróstegui I, Lafuente I, Vidaurreta I. Responsiveness and clinically important differences for the WOMAC and SF-36 after total knee replacement. Osteoarthritis Cartilage 2007;15:273-280

9] Bade MJ, Kohrt WM, Stevens-Lapsley JE. Outcomes before and after Total Knee Arthroplasty compared to healthy adults. J Orthop Sports Phys Ther 2010;40(9):559-567

10] Kittelson AL, Stackhouse SK, Stevens-Lapsley JE. Neuromuscular electrical stimulation after total joint arthroplasty: a critical review of recent controlled studies. Eur J Phys Rehabil Med 2013;49:909-920

11] Mintken PE, Carpenter KJ, Eckhoff D, Kohrt WM, Stevens JE. Early neuromuscular electrical stimulation to optimize quadriceps muscle function following total knee arthroplasty: a case report. J Orthop Sports Phys Ther 2007;37:364-371

12] Petterson S, Snyder-Mackler L. The use of neuromuscular electrical stimulation to improve activation deficits in a patient with chronic quadriceps strenght impairments following total knee arthroplasty. J Orthop Sports Phys Ther 2006;36:678-685

13] Gregory CM, Bickel CS. Recruitment patterns in human skeletal muscle during electrical stimulation. Phys Ther 2005;85:358-364 
14] Maffiuletti NA. Physiological and methodological considerations for the use of neuromuscular electrical stimulation. Eur J Appl Physiol 2010;110:223-234

15] Golaszewski S, Kremer C, Wagner M, Felber S, Aichner F, Dimitrijevic MM. Functional magnetic resonance imaging of the human motor cortex before and after whole-hand afferent electrical stimulation. Scand J Rehabil Med 1999;31:165-173

16] Stackhouse SK, Binder-Macleod SA, Stackhouse CA, McCarthy JJ, Prosser LA, Lee SCK et al. Neuromuscular electrical stimulation versus volitional isometric strength training in children with spastic Diplegic cerebral palsy: A preliminary study. Neurorehabil Neural Repair 2007;21:475-485.

17] Moher D, Shamseer L, Clarke M, Ghersi D, Liberati A, Petticrew M,et al. (PRISMA-P Group). Preferred reporting items for systematic review and meta-analysis protocols (PRISMA-P) 2015 statement. Syst Rev. 2015;1-4:1.

18] Neyeloff JL1, Fuchs SC, Moreira LB. Meta-analyses and Forest plots using a microsoft excel spreadsheet: step-by-step guide focusing on descriptive data analysis. BMC Res Notes. 2012;20-5:52.

19] Avramidis K, Strike PW, Taylor PN, Swain ID. Effectiveness of electric stimulation of the vastus medialis muscle in the rehabilitation of patient after total knee arthroplasty. Arch Phys Med Rehabil 2003;84:1850-1853

20] Avramidis K, Karachalios T, Popotonasios K, Sacorafas D, Papathanasiades AA, Malizos KN. Does electric stimulation of the vastus medialis muscle influence rehabilitation after total knee replacement? Orthopedics 2011;34(3):175-198

21] Levine M, McElroy K, Stakich V, Cicco J. Comparing conventional physical therapy rehabilitation with neuromuscular electrical stimulation after TKA. Orthopedics 2013;36(3):319-24.

22] Stevens-Lapsley JE, Balter JE, Wolfe P, Eckoff DG, Kohrt WM. Early neuromuscular electrical stimulation to improve quadriceps muscle strength after total knee arthroplasty: a randomized controlled trial. Phys Ther 2012;92:210-226

23] Petterson SC, Mizner RL, Stevens JE, Raisis L, Bodenstab A, Newcomb W, et al. Improved function from progressive strengthening interventions after total knee arthroplasty: a randomized clinical trial with an imbedded prospective cohort. Arthritis Rheum 2009;61(2):174-83

24] Demircioglu, D.T., Paker, N., Erbil, E., Bugdayci, D., Emre, T.Y. The effect of neuromuscular electrical stimulation on functional status and quality of life after knee arthroplasty: a randomized controlled study. J Phys Ther Sci 2015;27, 2501-2506. 
25] Stevens-Lapsley JE, Balter JE, Wolfe P, Eckhoff DG, Schwartz RS, Schenkman M, et al. Relationship between intensity of quadriceps muscle neuromuscular electrical stimulation and strength recovery after total knee arthroplasty. Phys Ther 2012;92(9):1187-96.

26] Laufer Y, Snyder-Mackler L. Response of male and female subjects after total knee arthroplasty to repeated neuromuscular electrical stimulation of the quadriceps femoris muscle. Am J Phys Med Rehabil 2010;89(6):464-472

27] Lewek M, Stevens J, Snyder-Mackler L. The use of electrical stimulation to increase quadriceps femoris muscle force in an elderly patient following a total knee arhtroplasty. Phys Ther 2001;81:1565-1571

28] Stevens J, Misner RL, Snyder-Mackler L. Neuromuscular electrical stimulation for quadriceps muscle strengthening after bilateral total knee arthroplasty: a case series. J Orthop Sports Phys Ther 2004;34(1): 21-29

29] Walls RJ, McHugh G, O'Gorman DJ, Moyna NM, O'Byrne JM. Effects of preoperative neuromuscular electrical stimulation on quadriceps strength and functional recovery in total knee arthroplasty. A pilot study. BMC Musculoskelet Disord. 2010;14-11:119 
Figure 1: The PRISMA flow diagram encapsulates the items assessed and the reasons for exclusions.

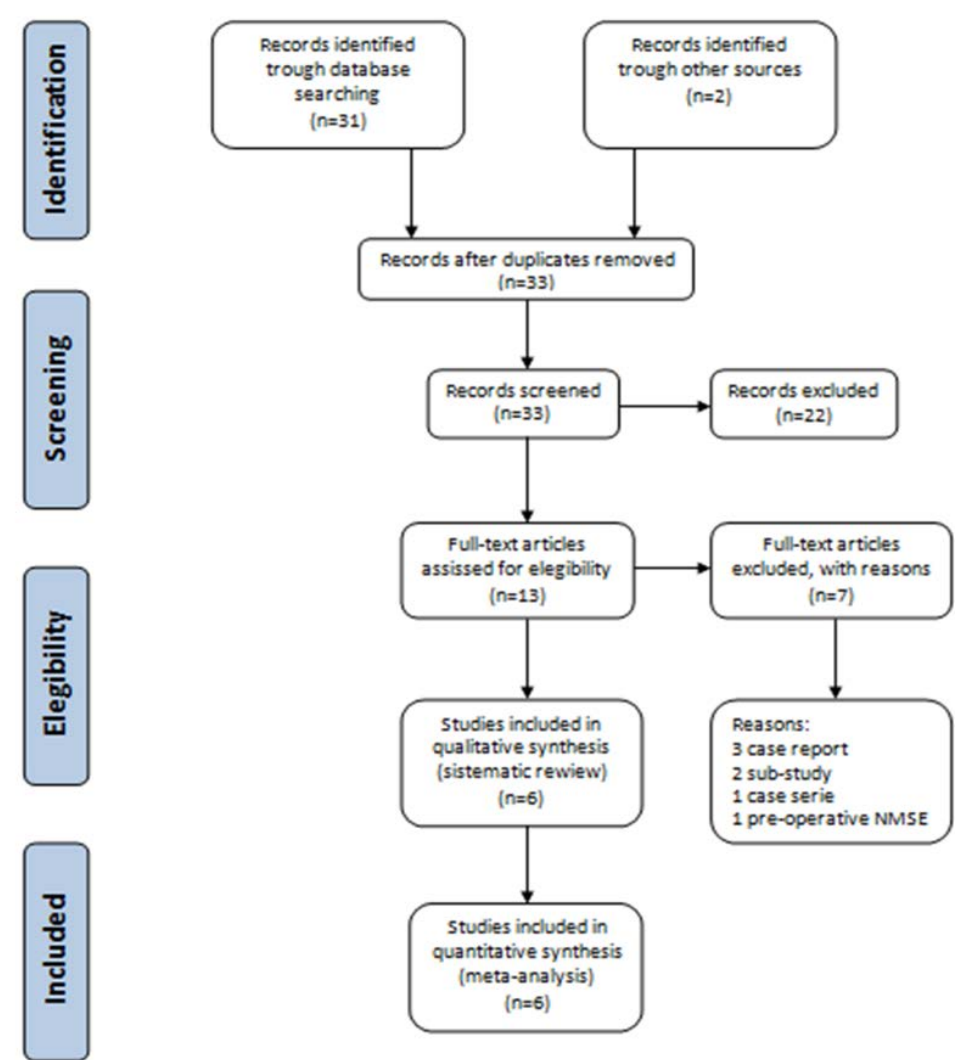


Figure 2: forest plot of the Mental Component Score of the SF-36 at 12 weeks $\left[1^{2}=\right.$ 60.11\% (Q statistics: 8.11, pvalue: 0.04, meaning a significant heterogeneity)]. Mean diff: mean difference; 95\% $\mathrm{Cl}$ : 95\% Confidence Interval; RE Model: Random-effect Model; NME: Neuromuscular electrical stimulation; SF-36: 36Item Short Form Health Survey.

MCS

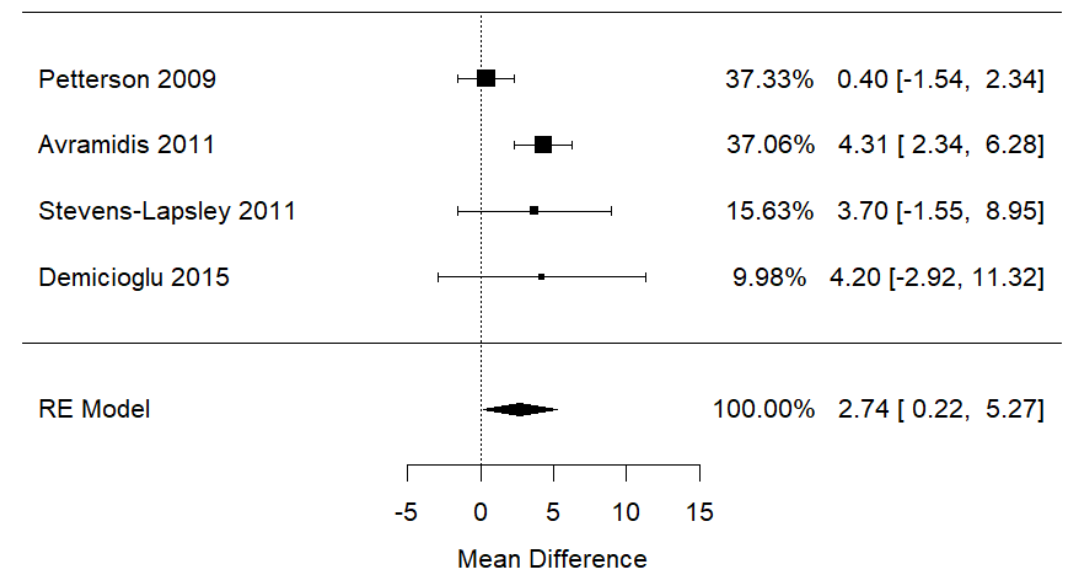


Figure 3: forest plot of the Physical Component Score of the SF-36 at 12 weeks [1

$=60.65 \%$ (Q statistics: 8.20, pvalue: 0.04, meaning a significant heterogeneity)]. Mean diff: mean difference; 95\% Cl: 95\% Confidence Interval; RE Model: Random-effect Model; NME: Neuromuscular electrical stimulation; SF-36: 36Item Short Form Health Survey.

PCS

\begin{tabular}{|c|c|c|c|c|c|c|}
\hline Petterson 2009 & & & $\because$ & & $30.15 \%$ & $-0.19[-3.12,2.74]$ \\
\hline Avramidis 2011 & & & $\mapsto$ & & $34.73 \%$ & $4.61[2.36,6.86]$ \\
\hline Stevens-Lapsley 2011 & & $\vdash$ & $\longrightarrow$ & & $24.06 \%$ & $0.10[-3.82,4.02]$ \\
\hline Dermicioglu 2015 & & & - & & $11.06 \%$ & $0.70[-6.71,8.11]$ \\
\hline RE Model & & & & & $100.00 \%$ & $1.65[-1.20,4.49]$ \\
\hline & $\Gamma$ & $T$ & $T$ & 7 & & \\
\hline & -10 & -5 & 5 & 10 & & \\
\hline
\end{tabular}


Table 1. Inclusion criteria

\begin{tabular}{|c|c|c|}
\hline & Inclusion criteria & exclusion criteria \\
\hline Patients & $\begin{array}{l}\text { patients of both sexes with at } \\
\text { east } 18 \text { years old who } \\
\text { inderwent unilateral PTG }\end{array}$ & $\begin{array}{l}\text { patients younger than } 18 \text { years } \\
\text { bilateral TKA }\end{array}$ \\
\hline ntervention & $\begin{array}{l}\text { traditional rehabilitation + post } \\
\text { pperatory NMES (4 weeks) }\end{array}$ & $\begin{array}{l}\text { pre-operatory NMES and not } \\
\text { ost-operatory }\end{array}$ \\
\hline Fomparator & traditional rehabilitation & \\
\hline Dutcome & $\begin{array}{l}\text { studies with clinical outcome } \\
\text { learly defined "a priori" }\end{array}$ & $\begin{array}{l}\text { studies without clinical } \\
\text { putcome clearly defined "a } \\
\text { priori" }\end{array}$ \\
\hline Study Design & Gontrolled randomized studies & $\begin{array}{l}\text { Controlled non-randomized } \\
\text { studies } \\
\text { Sub-studies } \\
\text { Systematic review } \\
\text { Case report } \\
\text { Case series } \\
\text { Editorials } \\
\text { Expert opinion }\end{array}$ \\
\hline
\end{tabular}


Table 2. Patients demographics: numerosity of the patients and demographic characteristics of the participants. The 2003 Avramidis study did not reported the BMI.

\begin{tabular}{|c|c|c|c|c|c|c|}
\hline & Avramidis ${ }^{19}$ & Petterson ${ }^{23}$ & $\begin{array}{l}\text { Stevens- } \\
\text { Lapsley }{ }^{22}\end{array}$ & Avramidis ${ }^{20}$ & Levine ${ }^{21}$ & pemircioglu ${ }^{24}$ \\
\hline $\begin{array}{l}\text { Number of } \\
\text { patients }\end{array}$ & 30 & 200 & 66 & 70 & 70 & 60 \\
\hline $\begin{array}{l}\text { NMES } \\
\text { reated/ } \\
\text { fontrols }\end{array}$ & $15 / 15$ & $100 / 100$ & $35 / 31$ & $35 / 35$ & $35 / 35$ & $30 / 30$ \\
\hline Female \% & $73 \%$ & $46 \%$ & $54,5 \%$ & $81,4 \%$ & $69,7 \%$ & $95 \%$ \\
\hline Age (mean) & 70 & 65 & 65 & 71 & 67 & 65 \\
\hline BMI (mean) & - & 29.8 & 29.1 & 27.3 & 31.2 & 29.6 \\
\hline
\end{tabular}


Table 3. Characteristics of the neuromuscular electrical stimulation sessions performed (NMES). Start of treatment: the day when the treatment was started after surgery. Modality of treatment: how many times NMES treatment was applied per day or per week. Duty cycle: ratio between the time when the machine dispenses the impulse and time of rest, expressed in seconds.

\begin{tabular}{|c|c|c|c|c|c|c|}
\hline NMES & Avramidis 19 & Petterson 23 & $\begin{array}{c}\text { Stevens- } \\
\text { Lapsley } 22\end{array}$ & Avramidis ${ }^{20}$ & Levine 21 & Demircioglu ${ }^{24}$ \\
\hline $\begin{array}{l}\text { Pre operative } \\
\text { reatment }\end{array}$ & & & & & 14 days & \\
\hline $\begin{array}{l}\text { tart of } \\
\text { reatment } \\
\text { days after } \\
\text { purgery) }\end{array}$ & 2 & 28 & 2 & 2 & 2 & 1 \\
\hline $\begin{array}{l}\text { Duration of } \\
\text { reatment } \\
\text { (minutes) }\end{array}$ & 120 & 15 & 15 & 120 & $20-30$ & 30 \\
\hline $\begin{array}{l}\text { Modality of } \\
\text { reatment }\end{array}$ & 2 times/ die & $\begin{array}{l}\text { 2-3 times/ } \\
\text { week }\end{array}$ & 2 times / die & 2 times / die & 1 time / die & 5 times/week \\
\hline Intensity & $\begin{array}{c}\text { Max } \\
\text { Tolerated }\end{array}$ & $\begin{array}{c}\text { Max } \\
\text { Tolerated }\end{array}$ & $\begin{array}{c}\text { Max } \\
\text { Tolerated }\end{array}$ & $\begin{array}{c}\text { Max } \\
\text { Tolerated }\end{array}$ & $\begin{array}{c}\text { Max } \\
\text { Tolerated }\end{array}$ & $\begin{array}{c}\text { Max } \\
\text { Tolerated }\end{array}$ \\
\hline $\begin{array}{l}\text { Frequency } \\
\mathrm{Hz})\end{array}$ & 40 & 50 & 50 & 40 & 75 & $30-100$ \\
\hline $\begin{array}{l}\text { Duty cycle } \\
\%)\end{array}$ & \begin{tabular}{|c|}
50 \\
$8 ”$ \\
ON/8”OFF
\end{tabular} & $\begin{array}{c}11 \\
10 ” O N / 80 ” O \\
\text { FF }\end{array}$ & \begin{tabular}{|c|}
25 \\
$15 ” O N / 45 ”$ \\
OFF
\end{tabular} & $\begin{array}{c}50 \\
\text { 8”ON/8”OF } \\
\text { F }\end{array}$ & $\begin{array}{c}29 \\
4 ” O N / 10 ” O \\
\text { FF }\end{array}$ & $\begin{array}{c}50 \\
0 " \mathrm{ON} / 10 \text { "OFF }\end{array}$ \\
\hline $\begin{array}{l}\text { Impulse } \\
\text { duration }(\mu \mathrm{s})\end{array}$ & 300 & 400 & 250 & 300 & 300 & 400 \\
\hline $\begin{array}{l}\text { Probe } \\
\text { dimensions } \\
\text { (cm) }\end{array}$ & $7 \times 7$ & $7.62 \times 12.7$ & $7.62 \times 12.70$ & $7.0 \times 7.0$ & $5.08 \times 10.16$ & $?$ \\
\hline
\end{tabular}


Table 4. Risk of bias The quality and risk of bias of individual randomized controlled trials included in the meta-analysis: + low risk; ? risk uncertain; - high risk

\begin{tabular}{|c|c|c|c|c|c|c|}
\hline & $\begin{array}{c}\text { Avramidis } \\
19\end{array}$ & $\begin{array}{c}\text { Petterson } \\
23\end{array}$ & \begin{tabular}{|c|} 
Stevens- \\
Lapsley \\
22
\end{tabular} & $\begin{array}{c}\text { Avramidis } \\
20\end{array}$ & $\begin{array}{c}\text { Levine } \\
21\end{array}$ & $\begin{array}{c}\text { pemirciogl } \\
\mathrm{u}^{24}\end{array}$ \\
\hline $\begin{array}{l}\text { Generation of a random } \\
\text { sequence (Bias of } \\
\text { selection) }\end{array}$ & + & + & + & + & + & + \\
\hline $\begin{array}{l}\text { Blind allocation } \\
\text { (Bias of selection) }\end{array}$ & ? & $?$ & + & ? & $?$ & + \\
\hline $\begin{array}{l}\text { Blind criteria for } \\
\text { participants } \\
\text { (bias of participation) }\end{array}$ & ? & + & ? & ? & ? & ? \\
\hline \begin{tabular}{|l} 
Blind criteria for \\
evaluators \\
(Bias of survey)
\end{tabular} & - & + & - & + & ? & ? \\
\hline $\begin{array}{l}\text { Incomplete data } \\
\text { (Bias of friction) }\end{array}$ & + & + & + & + & + & + \\
\hline $\begin{array}{l}\text { Fundings } \\
\text { (fundings Bias) }\end{array}$ & + & + & - & + & + & + \\
\hline
\end{tabular}


Table 5. Evaluated clinical outcomes for the selected studies.

Physical performance. VAS: visual analog scale; TUG: Test Up and Go (a simple test used to evaluate the mobility of the subject: measures the time to get up from a chair, walk three meters, turn around, return to the chair and sit down again); AROM: active range of motion; PROM: passive range of motion; WST: walking speed test; 3MWT: 3-minute walking test; 6MWT: 6-minute walking test (measure the distance that the subject covers walking for 3 or 6 minutes, respectively); SCT: Stair Climbing Test (measures the time to go up and down 12 steps of a stair); PCI: physiological cost index

Knee Functionality. KSS: Knee Society Score, WOMAC: Western Ontario and McMaster Universities; OXFORD: Oxford 12 items knee score; GRS: global rating score; KOS-ADLS: Knee Outcome Survey - Activities of Daily Living Scale; HSS: Hospital for Special Surgery Knee Score; SF-36: sf-36 italian version

\begin{tabular}{|c|c|c|c|c|c|c|}
\hline & $\begin{array}{c}\text { Avramidi } \\
\mathrm{s}^{19}\end{array}$ & $\begin{array}{c}\text { Petterson } \\
23\end{array}$ & $\begin{array}{l}\text { Stevens- } \\
\text { Lapsley } 22\end{array}$ & $\begin{array}{c}\text { Avramidis } \\
20\end{array}$ & Levine ${ }^{21}$ & $\begin{array}{c}\text { Demirciogl } \\
\mathrm{u}^{24}\end{array}$ \\
\hline \multicolumn{7}{|l|}{ Physical performance. } \\
\hline VAS & & & & & & $\mathrm{X}$ \\
\hline TUG & & $\mathrm{X}$ & $\mathrm{X}$ & & $\mathrm{X}$ & $\mathrm{X}$ \\
\hline AROM & & & $\mathrm{X}$ & & $\mathrm{X}$ & \\
\hline PROM & & & & & & $\mathrm{X}$ \\
\hline WST & & & & $\mathrm{X}$ & & \\
\hline 3MWT & $\mathrm{X}$ & & & & & \\
\hline $6 \mathrm{MWT}$ & & $\mathrm{X}$ & $\mathrm{X}$ & & & \\
\hline Quadriceps strength & & $\mathrm{X}$ & $\mathrm{X}$ & & & \\
\hline Quadriceps activation & & $\mathrm{X}$ & $\mathrm{X}$ & & & \\
\hline Posterior tight strength & & & $\mathrm{X}$ & & & \\
\hline SCT & & $\mathrm{X}$ & $\mathrm{X}$ & & & \\
\hline PCI & $\mathrm{X}$ & & & $\mathrm{X}$ & & \\
\hline \multicolumn{7}{|l|}{ Knee Functionality. } \\
\hline KSS & & & & & $\mathrm{X}$ & \\
\hline WOMAC & & & $\mathrm{X}$ & & $\mathrm{X}$ & $\mathrm{X}$ \\
\hline OXFORD & & & & $\mathrm{X}$ & & \\
\hline GRS & & & $\mathrm{X}$ & & & \\
\hline KOS-ADLS & & $\mathrm{X}$ & & & & \\
\hline HSS & $\mathrm{X}$ & & & & & \\
\hline SF-36 & & $\mathrm{X}$ & $\mathrm{X}$ & $\mathrm{X}$ & & $\mathrm{X}$ \\
\hline
\end{tabular}

